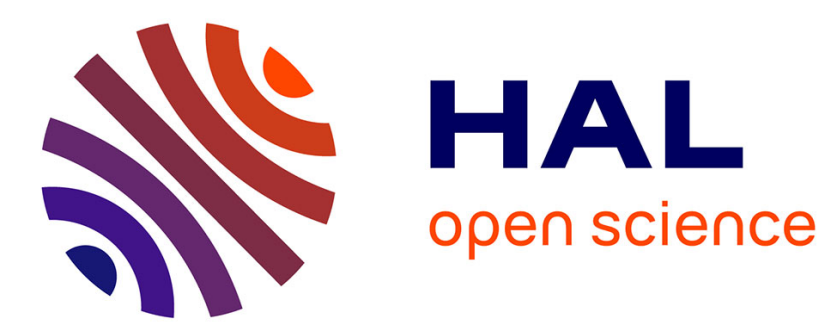

\title{
Three-dimensional visualization and physical properties of dendrites in thin samples of a hexagonal columnar liquid crystal
}

\author{
J. Géminard, P. Oswald
}

\section{- To cite this version:}

J. Géminard, P. Oswald. Three-dimensional visualization and physical properties of dendrites in thin samples of a hexagonal columnar liquid crystal. Journal de Physique II, 1994, 4 (6), pp.959-974. 10.1051/jp2:1994177 · jpa-00248019

\section{HAL Id: jpa-00248019 https://hal.science/jpa-00248019}

Submitted on 1 Jan 1994

HAL is a multi-disciplinary open access archive for the deposit and dissemination of scientific research documents, whether they are published or not. The documents may come from teaching and research institutions in France or abroad, or from public or private research centers.
L'archive ouverte pluridisciplinaire HAL, est destinée au dépôt et à la diffusion de documents scientifiques de niveau recherche, publiés ou non, émanant des établissements d'enseignement et de recherche français ou étrangers, des laboratoires publics ou privés. 
Classification

Physics Abstracts

$68.70-64.70 \mathrm{M}-61.50 \mathrm{C}$

\title{
Three-dimensional visualization and physical properties of dendrites in thin samples of a hexagonal columnar liquid crystal
}

\author{
J. C. Géminard and P. Oswald \\ Laboratoire de Physique, Ecole Normale Supérieure de Lyon, 46 Allée d'Italıe, 69364 Lyon \\ Cedex 07, France
}

(Recelved 22 November 1993, accepted in final form 7 March 1994)

\begin{abstract}
Résumé. - Mettant à profit la biréfringence de la mésophase colonnaire hexagonale de l'hexaoctyloxytriphénylène, nous montrons qu'il existe. en échantillon mince, deux types de dendrites en fonction du rapport de la longueur de diffusion $\ell_{\mathrm{d}}$ à l'épaisseur $d$ de l'échantillon. Lorsque $\ell_{\mathrm{d}} / d>50$, les dendrites sont $\langle 2 \mathrm{D} »$ et remplissent la totalité de l'épaisseur de l'échantillon. Lorsque $\ell_{\mathrm{d}} / d<10$, les dendrites sont « $3 \mathrm{D}$ » et un film de liquide isotrope les sépare des surfaces qui limitent l'échantillon. Les dendrites « $2 \mathrm{D} \gg$ vérifient la relation d'Ivantsov et ont une constante de stabilité $\sigma_{2 \mathrm{D}}^{*} \approx 0,041$ indépendante de l'épaisseur de l'échantillon. Par contre, bien qu'elles ne vérifient jamais la loi d'Ivantsov. les dendrites «3D » ont une constante de stabilité indépendante de l'épaisseur $\sigma_{3 \mathrm{D}}^{*} \approx 0,005$ lorsque leur vitesse est assez grande $\left(\ell_{\mathrm{d}} / d<1\right)$.
\end{abstract}

\begin{abstract}
Using the optical birefringence of the hexagonal columnar mesophase of the hexaoctyloxytriphenylene, we show that two types of stationary dendrites exist in thin samples, depending on the ratio of the diffusion length $\ell_{d}$ over the sample thickness $d$. Either $\ell_{\mathrm{d}} / d>50$ and the dendrites are $42 \mathrm{D} \gg$ and fill the whole sample thickness. or $\ell_{\mathrm{d}} / d<10$ and they are « 3D » and separated from the limiting surfaces by a film of isotropic liquid. The 2D-dendrites satisfy the Ivantsov relation and have a stability constant $\sigma_{2 \mathrm{D}}^{*} \approx 0.041$ independent of the sample thickness. By contrast and surprisingly, when their velocity is large enough $\left(\ell_{\mathrm{d}} / d<1\right)$, the 3Ddendrites have an apparent stability constant $\sigma_{3 \mathrm{D}}^{*} \approx 0.005$ independent of the thickness even if they do not verify the Ivantsov relation.
\end{abstract}

\section{Introduction.}

The problem of the dendritic gowth of an alloy has recently attracted much interest and effort from experimentalists. This is mainly due to the discovery of the "microscopic solvability " principle according to which dendrite velocity and growth directions are selected by the surface energy anisotropy [1]. More precisely, stationary dendrites can only grow along crystallographic directions of minimal surface energy (anisotropy $\varepsilon$ ) with a stability constant, $\sigma=2 D_{\mathrm{L}} d_{0}^{\mathrm{c}} / \rho^{2} V$, that only depends on $\varepsilon$. The parameters $\rho$ and $\mathrm{V}$ are, respectively, the radius of curvature and the velocity of the tip of the dendrite, $D_{\mathrm{L}}$ is the solute diffusion coefficient in the liquid and $d_{0}^{\mathrm{c}}$ the chemical capillary length. In order to test these theoretical 
predictions, several experiments have been recently performed with transparent materials such as organic plastic crystals (succinonitrile and pivalic acid) of inorganic salts $\left(\mathrm{NH}_{4} \mathrm{Br}\right)$. The main results of these experiments, gathered in two recent review articles [2], show that the theory-experiment agreement is only qualitative, even if there is now no doubt that anisotropy plays a central role during dendritic growth. The discrepancy between theory and experiments may be explained by the fact that the problems are still numerous, for example experimentally for the measurement of the surface energy anisotropy $\varepsilon$, and theoretically to take into account the lack of axisymmetry of real three-dimensional dendrites [3]. Another problem, not mentioned by Gollub but crucial experimentally, concerns the confinement of the dendrites due to the finite thickness of the samples. In general, it is admitted that dendrites are free when the diffusion length $\ell_{\mathrm{d}}=D_{\mathrm{L}} / V$ is smaller than the sample thickness ( $d$ in the following). This affirmation is based on the fact that, experimentally, the product $\rho^{2} V$ is found to be constant when $\ell_{\mathrm{d}}<d$ [4]. In the following, we show by experiments performed in thin samples that this criterion must be reconsidered and that the confinement effects may be important even if the product $\rho^{2} V$ is found to be constant.

In order to tackle this problem, we have chosen to study a discotic liquid crystal, the hexaoctyloxytriphenylene. The pure compound has a hexagonal columnar-isotropic phase transition at $86.1^{\circ} \mathrm{C}$. It has several advantages over classical materials : first, it orients spontaneously in homeotropic anchoring in thin samples (i.e. with the columns perpendicular to the glass plates) ; second, its physical constants (surface tension, anisotropy, diffusivities and kinetic coefficient) and its phase diagram are known [5-7]; third, it is very easy to grow dendrites [5a, 6] ; finally, it is birefringent, which allows us to visualize the three-dimensional structure of growing germs.

The plan of the article is as follows. In section 2, we describe the experimental set-up and the optical method of three-dimensional visualization of the germs. In section 3, we focus on the transient regimes which lead to dendrites and on global properties of growing germs. Finally, properties of $2 \mathrm{D}$ - and $3 \mathrm{D}$-dendrites are given in section 4 .

\section{The experimental procedure.}

In order to observe the free growth of the hexagonal mesophase, we sandwich a heated droplet of isotropic liquid between two square glass plates $(20 \times 20 \times 1 \mathrm{~mm})$. The thickness of the liquid cristal layer, which ranges from $2 \mu \mathrm{m}$ to $20 \mu \mathrm{m}$, is measured by conoscopy to about $\pm 0.1 \mu \mathrm{m}$ on an optical bench equipped with a L.A.S.E.R. and a Brace-Köhler compensator [9]. This method is usable because the liquid crystal is birefringent.

The sample is then placed into an oven in which nitrogen circulates. This precaution avoids a too fast degradation of the liquid crystal in contact with air $\left(\approx 0.01{ }^{\circ} \mathrm{C} / \mathrm{h}\right)$. The temperature is controlled to $\pm 3 \mathrm{mK}$ with a temperature controller A.T.N.E. A.T.S.R. 100 and is homogeneous within $10 \mathrm{mK}$ over the whole surface of the sample. The sample can be moved inside the oven from outside with the help of a $X-Y$ translation stage, which makes it possible to observe its whole surface through a microscope.

The experimental procedure to grow a germ at a given supersaturation is the following : first, the solidified sample is slowly heated until in its isotropic phase $\left(1^{\circ} \mathrm{C} / \mathrm{h}\right.$ typically). This allows us to measure the solidus and liquidus temperatures ( $T_{1}$ and $T_{\mathrm{s}}$ respectively) and to know where we are in the phase diagram (Fig. 1). Then, the sample is quickly undercooled at the chosen temperature $T$. The quench method, described in a previous article [7], allows us to reach the gowth temperature in a few seconds, before the first germs nucleate. It can happen that no germ nucleates because the chosen supersaturation is too small $(\Delta<0.15)$. In this case, we do not melt completly the hexagonal mesophase in order to isolate and equilibrate a small germ (typically $10 \mu \mathrm{m}$ ) which is then cooled down at the chosen temperature. 


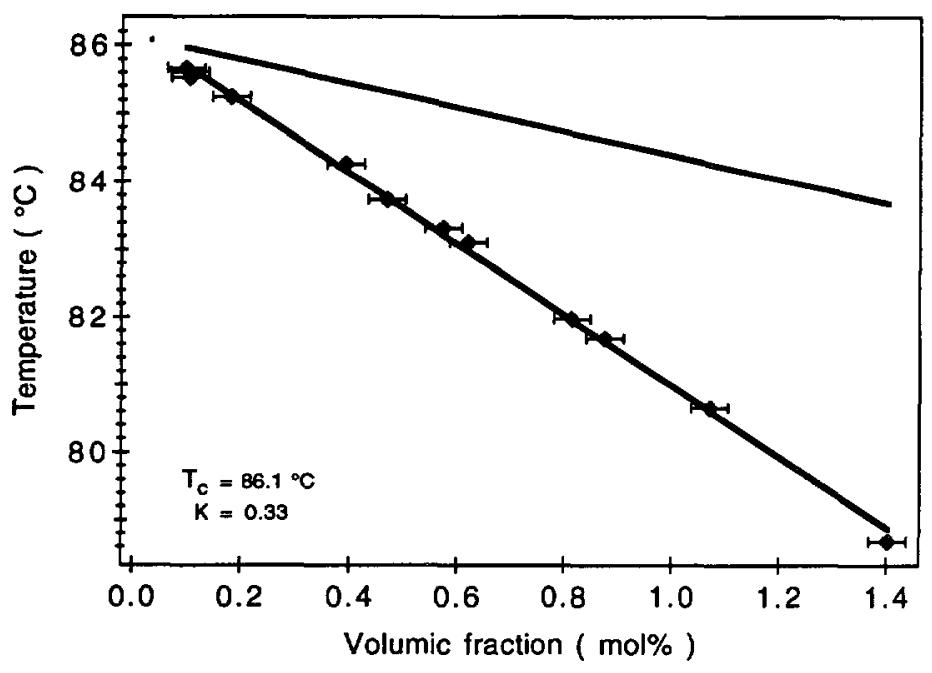

Fig. 1. - Experimental phase diagram. It has been determined as in reference [5a]. Impurity concentration (in mol\%) has been estimated from the Vant'Hoff law.

When the germ is growing, it does not fill necessarily the whole sample thickness $d$. In order to measure its local thickness $d_{\mathrm{m}}$, we have observed the germ between crossed polarizers after opening the aperture diaphragm of the microscope. Under these conditions, the isotropic phase is dark (intensity $I_{0}$ ) whereas the columnar mesophase appears to be gray (intensity $I>I_{0}$ ). On the pictures, the local intensity $I$ depends on the local value of $d_{\mathrm{m}}$ and saturates towards $I_{1}$ when $d_{\mathrm{m}}=d$. Let $d_{\mathrm{r}}$ be the relative thickness of the mesophase $\left(d_{\mathrm{r}}=d_{\mathrm{m}} / d\right)$. Using the optical bench, we have shown experimentally [9] that

$$
d_{\mathrm{r}}=\sqrt{\frac{I-I_{0}}{I_{1}-I_{0}}}
$$

provided that the sample is thin enough $(d<20 \mu \mathrm{m})$. In this formula, $I, I_{0}$ and $I_{1}$ can be measured simultaneously if the germ touches the two limiting glass plates in its center ; if not, $I_{1}$ must be mesured later after complete crystallization of the sample. This time lag between the measurements introduces systematic errors (of a few \%) which are due to the fluctuations of the used Hg-light source. Another limitation stems from the fact that this optical method is not applicable nearby the vertical part of the interface because the rays are too strongly deviated in this region (Becke fringes). Experimentally, the optical intensity $I$ is measured with a computer-assisted imaging system composed of a CCD Black \& White Video Camera (Panasonic WV-BL200/G), of a Video Cassette Recorder (Panasonic AG 6720) and of an Apple Macintosh Quadra 700 equipped with a Frame Grabber Card (Data Translation Quick Capture DT2255).

In order to test this optical method and to validate the phase diagram of figure 1 , we have measured the volumic fraction of solid $n_{\mathrm{s}}$ at equilibrium as a function of the chemical supersaturation $\Delta_{c}$ defined to be

$$
\Delta_{\mathrm{c}}=\frac{T_{\mathrm{l}}-T}{\left(T_{\mathrm{c}}-T\right)(1-K)}
$$




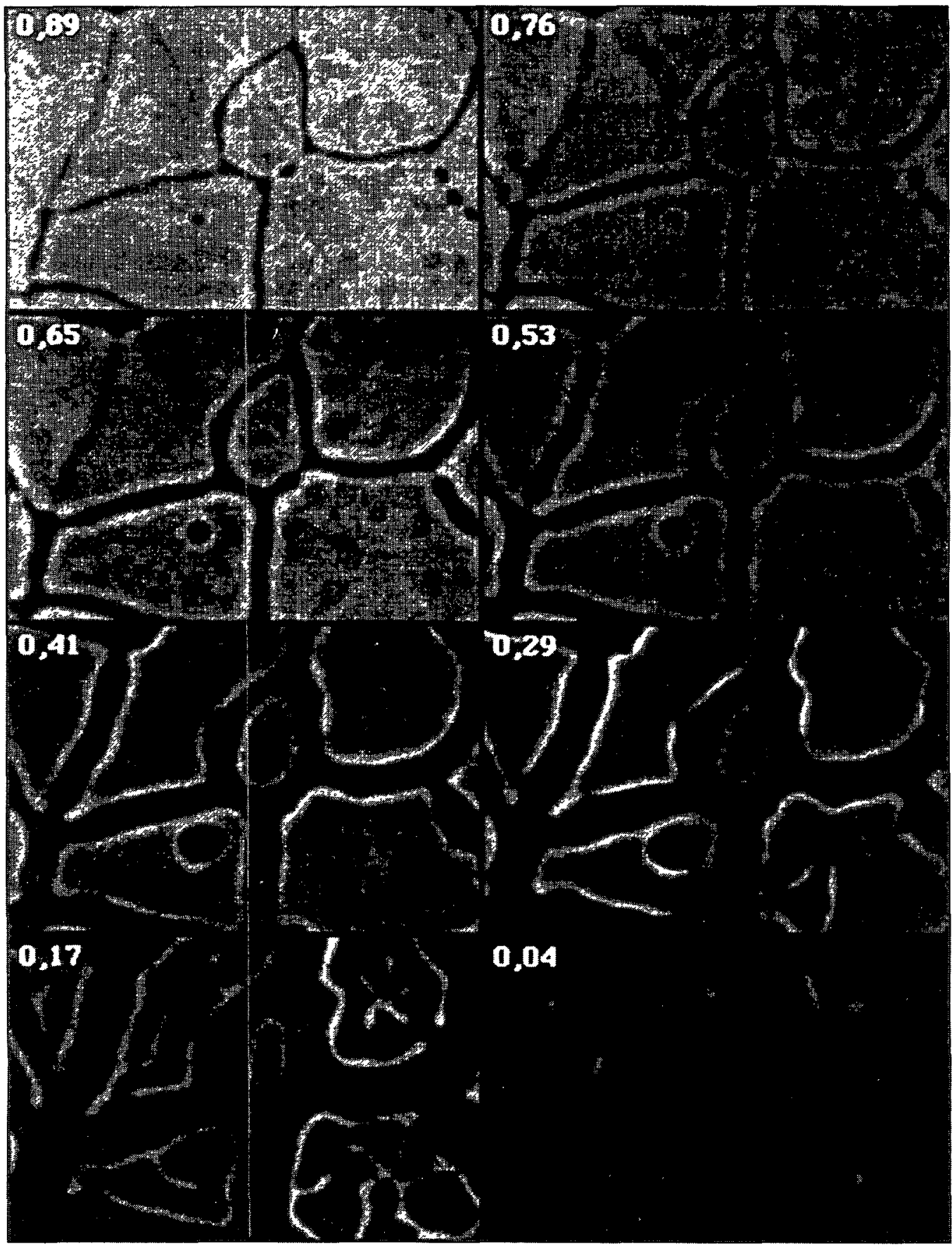

a)

Fig. 2. - a) Sample at equilibrium photographed between crossed polarizers at various supersaturations (indicated on each picture). Note that the intensity is not homogeneous within each domain which means that there is liquid between the solid and the two glass plates. The sample thickness is $d \approx 10 \mu \mathrm{m}$. b) Volumic fraction of solid $n_{\mathrm{b}}(\bullet)$ versus supersaturation $\Delta_{\mathrm{c}}$. We also reported the apparent solid fraction $(+)$ which is obtained by only measuring the germ area. This measurement shows the importance to take into account the hidden liquid as long as $\Delta_{c}>0.3$. 


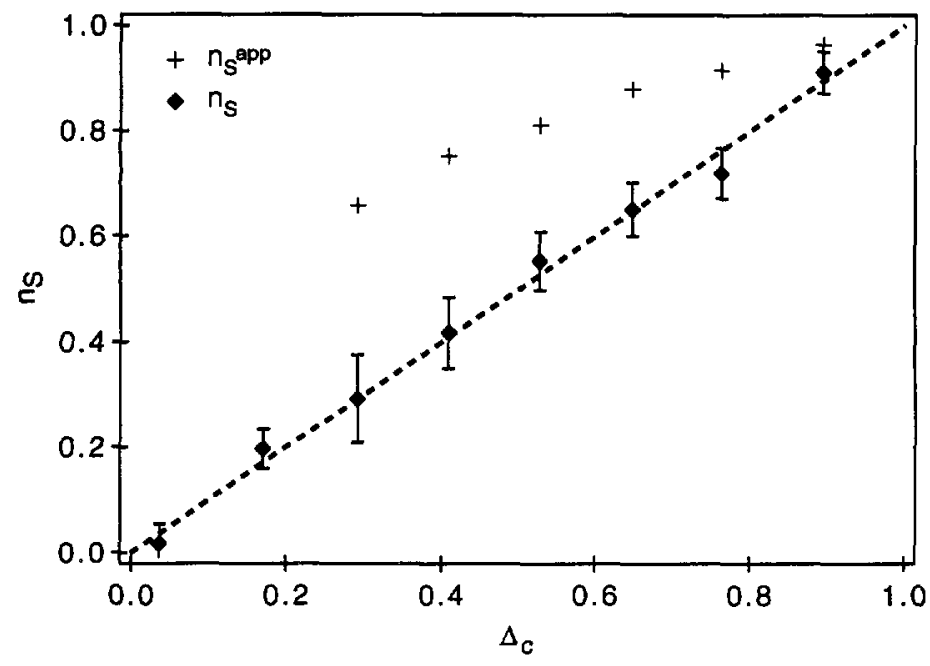

b)

Fig. 2 (continued).

where $T$ is the temperature $\left(T_{\mathrm{s}}<T<T_{1}\right), K$ the impurity partition coefficient $(K \approx 0.33)$ and $T_{\mathrm{c}}=86.1{ }^{\circ} \mathrm{C}$ the transition temperature of the pure compound. It is easy to show that at equilibrium $n_{\mathrm{s}}=\Delta_{\mathrm{c}}$. In order to test this relation, we have measured $n_{\mathrm{s}}$ at different supersaturations. This quantity is obtained by integrating $d_{\mathrm{r}}$ (itself obtained from Eq. (1)) over a surface of unit area (Fig. 2a). At each temperature, the system is held at constant temperature during a time which is at least 10 times longer than the diffusion time across the biggest solid grain. The data reported in figure $2 \mathrm{~b}$ show that $n_{\mathrm{s}}=\Delta_{\mathrm{c}}$ with a very good accuracy. This result validates the methods of measurement of both the phase diagram and of the chemical supersaturation $\Delta_{\mathrm{c}}$.

In the following section, we describe the transient regimes which lead to stationary dendrites.

\section{Global properties of germs in the transient predendritic regime.}

So far, all our experiments in the dendritic regime were performed in very thin samples ( $d$ of the order of a few $\mu \mathrm{m})$ and at small supersaturation $\left(\Delta_{c}<0.4\right)$. In this case, we found that the radius of destabilization of an initially circular germ is in good agreement with the MullinsSekerka theoretical predictions [5b]. We also observed that the time evolution of the germ surface area is linear without slope variation at the destabilization [6], in agreement with the numerical simulations of Brush and Sekerka [10]. Finally, we observed that after a transient regime, called petal-shape regime, stationary dendrites form with a well-defined stability constant $[5 a, 6]$.

In all these experiments, we implicitly assumed that three-dimensional effects were negligible because both the diffusion length and the radius of curvature at the tip of the dendrites were much larger than the thickness. In this section and in the following ones, we reconsider this hypothesis and systematically analyze the thickness effects, first in thick samples $(7-15 \mu \mathrm{m})$ and then in very thin ones $(\approx 2 \mu \mathrm{m})$. 
3.1 « THICK 》 SAMPLE $(7 \mu \mathrm{m}<d<15 \mu \mathrm{m})$. - Figure 3 shows the typical evolution at $\Delta_{c}=0.1$ of a circular germ in a $9.2 \mu \mathrm{m}$-thick sample observed between crossed polarizers. At the beginning $\left(T \approx T_{1}\right)$, the germ is at equilibrium and fills the whole thickness. When the temperature is abruptly decreased, the germ starts growing while remaining circular; on the other hand, its optical contrast changes (it becomes darker on the sides) which means that its thickness locally decreases. That also means that the germ becomes 3D and first destabilizes in the sample thickness (Fig. 3a). It then destabilizes in the sample plane by forming six petals (Fig. 3b) which partly fill the sample thickness. Each petal then grows by slowing down and by thickening on the sides as the white strip surrounding the germ shows. In figure $3 \mathrm{f}$, the germ has everywhere resticked the two glass plates (see also Fig. $3 \mathrm{~g}$ ). From this time, each petal becomes a two-dimensional dendrite similar to that studied in reference [5a] and in the following section. The passage 3D-2D is particularly visible by plotting the germ surface area and the radius of its circular envelope as a function of time (Fig. 4). On this two graphs, the slope discontinuities mark respectively the petal-dendrite transition and 3D-2D passage.

The germ evolution becomes different when the supersaturation is larger than 0.3 (in the same $9.2 \mu \mathrm{m}$-thick sample). Figure 5 shows the example of a germ growing at $\Delta=0.4$. At this supersaturation, the six branches of the germ never rejoin the two glass plates and lead to $3 \mathrm{D}$ dendrites which are separated from the two glass plates by a thin liquid film. The relative thickness of dendrites at this supersaturation is constant and is equal to 0.78. Figure 6 shows global properties of this germ. As in the previous case, it clearly appears by plotting the time evolution of the germ radius (measured at the tip of the primary dendrites, Fig. 6a) that two growth regimes must be distinguished: the petal-shape regime during which the growth velocity of each petal decreases and the dendritic regime characterized by a constant growth rate. In this stationary regime, each dendrite is $3 \mathrm{D}$ with well-defined velocity and tip radius of curvature. On the other hand, the time evolution of the surface area $A$ of the germ is qualitatively different from what we observed in the previous case (Fig. 6b) : indeed, $A$ is no longer linear in time and there is no slope variation at the petal-dendrite transition, the growth remaining $3 \mathrm{D}$ in the dendritic regime. Finally, we have plotted in figure $6 \mathrm{c}$ the apparent and the real volumic fractions of solid (respectively $n_{\mathrm{s}}^{\text {app }}$ and $n_{\mathrm{s}}$ ) contained within the hexagonal envelope of the germ. These two quantities decrease in the petal-shape regime and tend towards two different constants in the dendritic regime. It is worth noting that $n_{\mathrm{s}}$ tends to $\Delta_{\mathrm{c}}$ in agreement with the impurity conservation law in a stationary regime while $n_{\mathrm{s}}^{\text {app }}$ saturates to a larger value given by $n_{\mathrm{s}}^{\text {app }}=n_{\mathrm{s}} /\left\langle d_{\mathrm{r}}\right\rangle$ where $\left\langle d_{\mathrm{r}}\right\rangle$ is the average relative thickness of the germ $\left(\left\langle d_{\mathrm{r}}\right\rangle \approx 0.78\right.$ in this example).

Let us now describe what happens in a very thin sample.

3.2 « THIN » SAMPLE $(d<5 \mu \mathrm{m})$. - The thinner the samples, the more difficult the visualization of the 3D-effects. This is only due to the lack of optical contrast on the pictures when the liquid crystal layer is thin. In spite of these difficulties, we performed similar experiments in a $2 \mu \mathrm{m}$-thick sample, and we observed that, in such a sample, 3D effects are not visible through the microscope at very small supersaturation $\left(\Delta_{c} \approx 0.1\right)$, even during the transient petal-shape regime during which the growth rate decreases (Fig. 7a). This is confirmed by the measurement of the evolution law of the germ surface area as a function of time (Fig. 7b). Contrary to what happens in thick samples (see Fig. 6b), there is no noticeable variation of the slope $\mathrm{d} A / \mathrm{d} t[5,6]$, which means that the growth process is $2 \mathrm{D}$ during all the experiment, even before the germ destabilizes in its basal plane. This behavior remains unchanged as long as $\Delta_{c}$ is small (typically $\Delta_{c}<0.3$ ).

The situation is different at large supersaturation (typically when $\Delta_{c}>0.5$ ). The germ behavior is then similar to what happens in thick samples with formation of $3 \mathrm{D}$-dendrites. In this case, the envelope of the germ is hexagonal and $n_{\mathrm{s}}^{\text {app }}$ is clearly larger than the imposed 

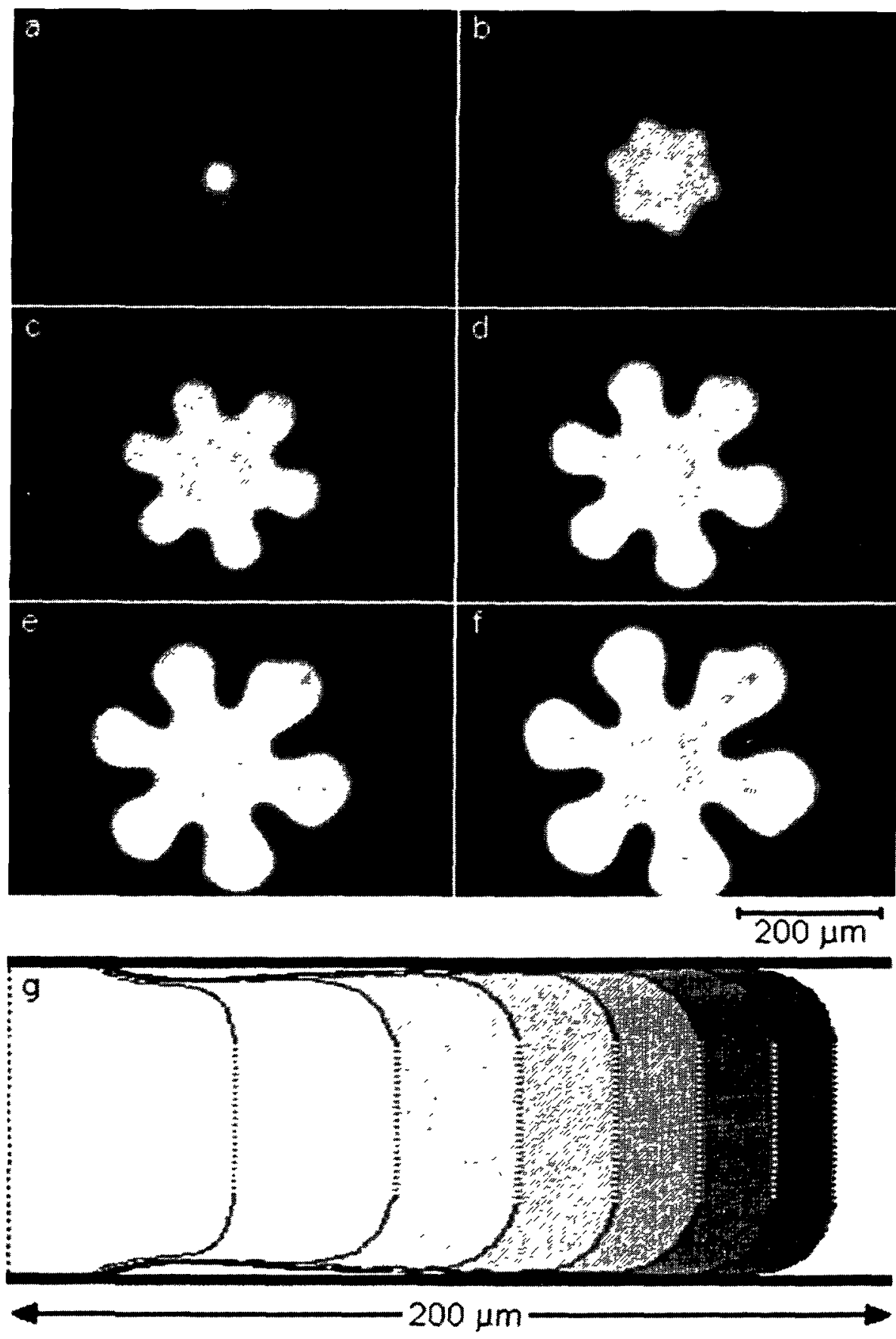

Fig. 3. - Growth of an initially circular germ at $\Delta_{c}=0.1\left(d=9.2 \mu \mathrm{m}\right.$ and $\left.\Delta T=6^{\circ}\right)$. At the beginning, the germ touches the two glass plates. It first destabilizes in the sample thickness (a) before developing a hexagonal modulation in the horizontal plane (b). In ( $f$ ) the six petals touch the glass plates on the sides. The time interval between two photographs is $10 \mathrm{~min}$. In $(\mathrm{g})$ we show the time evolution of the transverse section of a petal taken along the growth direction. The time interval between two profiles is $10 \mathrm{~min}$. 


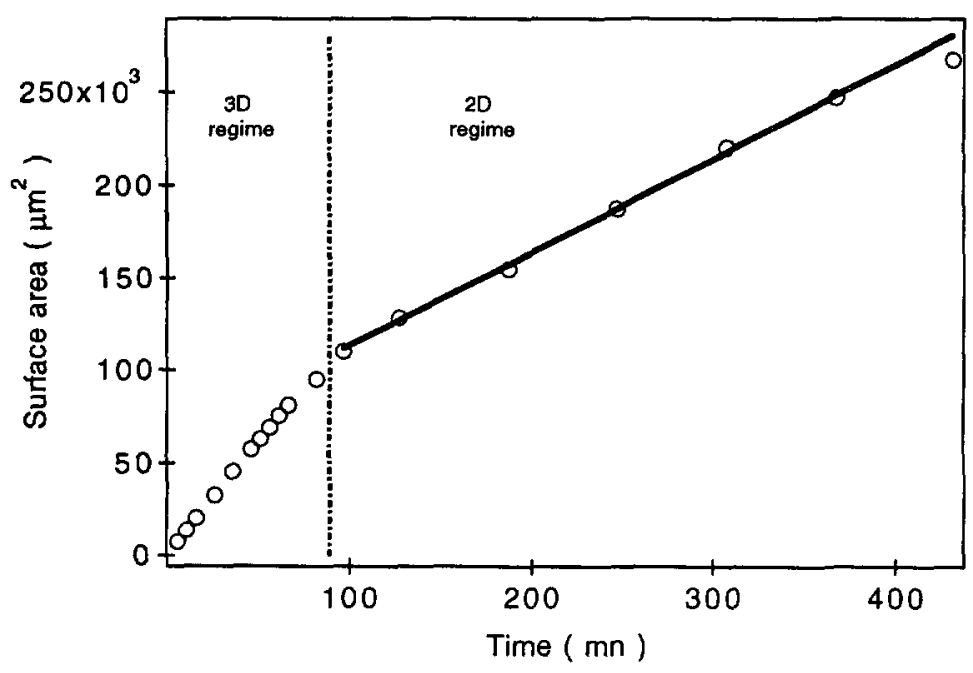

a)

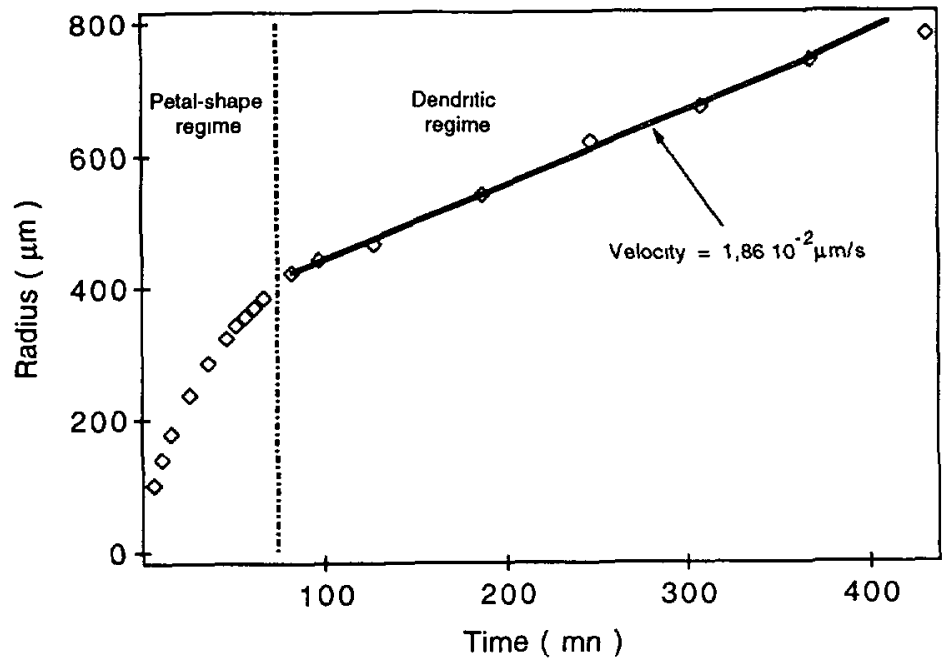

b)

Fig. 4. - a) Surface area of the germ of figure 3 versus time $\left(\Delta_{c}=0.1, d=9.2 \mu \mathrm{m}\right)$. b) Radius of its circular envelope versus tıme.

supersaturation $\Delta_{c}$. This means that, even in very thin samples, there is a liquid film between the solid and the limiting glass plates.

The main conclusion of this section is that there exists a critical supersaturation $\Delta_{c}^{*}$ below which the dendrites are 2D (i.e. touch the two glass plates) whereas they are 3D above (i.e. separated from the two glass plates by a liquid film). This supersaturation does not change a lot with the sample thickness and typically ranges between 0.3 (for thick samples) and 0.5 (for thin samples). In the following section, we focus on physical properties of stationary dendrites. 


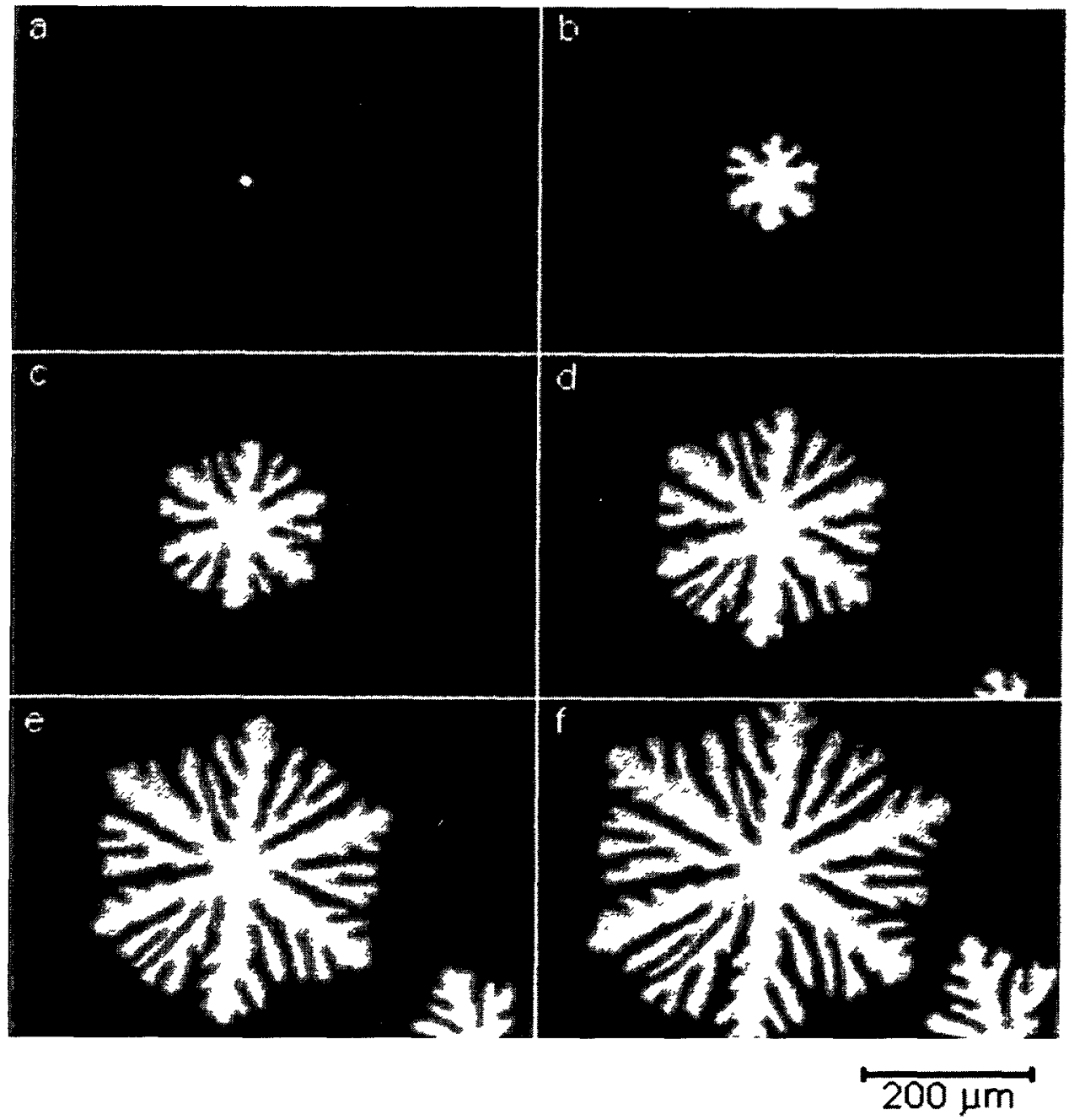

Fig. 5. - Germ at $\Delta_{\mathrm{c}}=0.4$ photographed between crossed polarizers. The time interval between two photographs is $15 \mathrm{~s}$.

\section{Physical properties of stationary dendrites.}

We have shown experimentally the existence of two types of dendrite. In this section, we give their main properties in the stationary regime while insisting on the finite thickness effects.

A dendrite is characterized by its two radii of curvature at the tip and its velocity. Experimentally, we are only able to measure the velocity $V$ and the radius of curvature in the plane of the sample $\rho$. Because of the narrow size of the region that can be fitted by a parabola, this last measurement is delicate and noisy, particularly at large supersaturations. Indead, the side-branches appear closer and closer to the tip when $\Delta_{c}$ increases. An example of parabolic fit is shown in figure 8 . The velocity is much easier to measure thanks to the Video Cassette Recorder.

Thanks to the optical measurement of the relative thickness of the crystal and the measurements of $V$ and $\rho$, we have analyzed the two types of dendrite separately. 


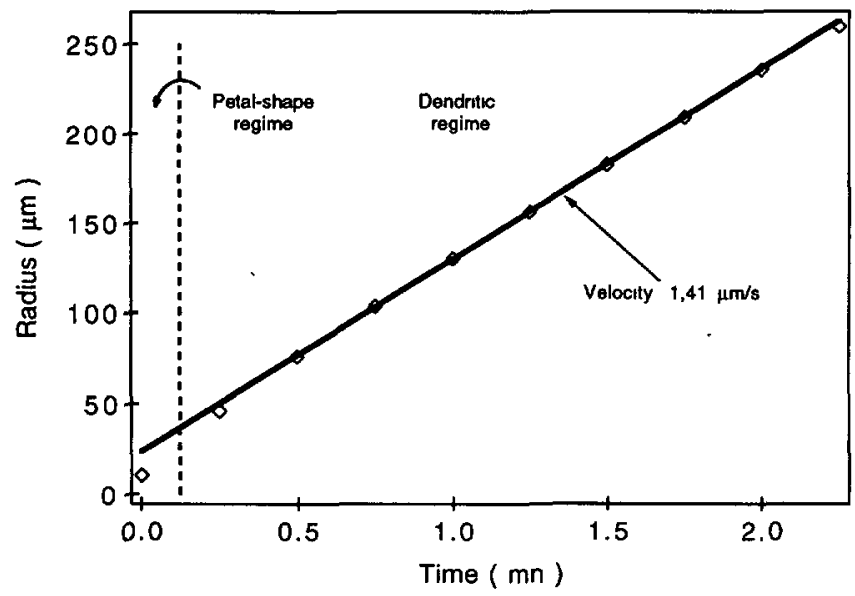

a)

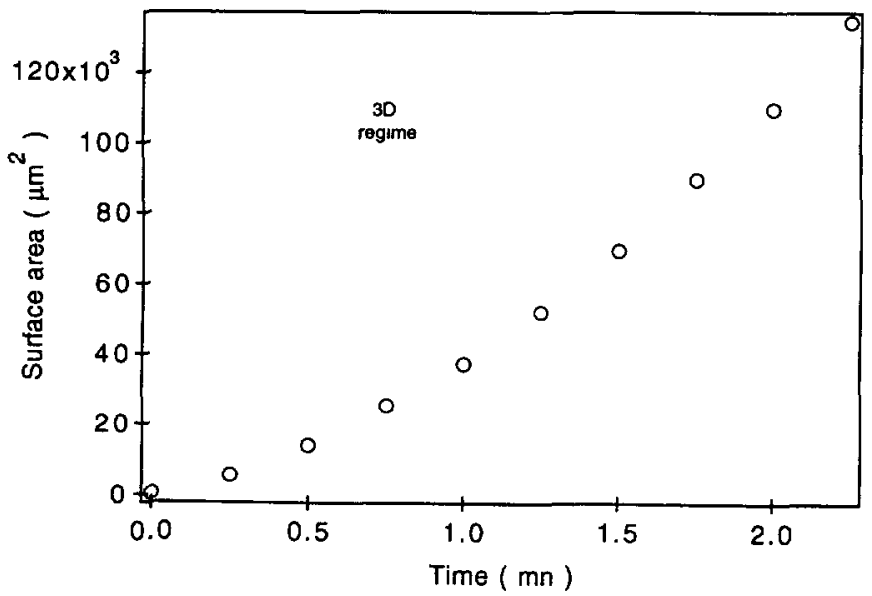

b)

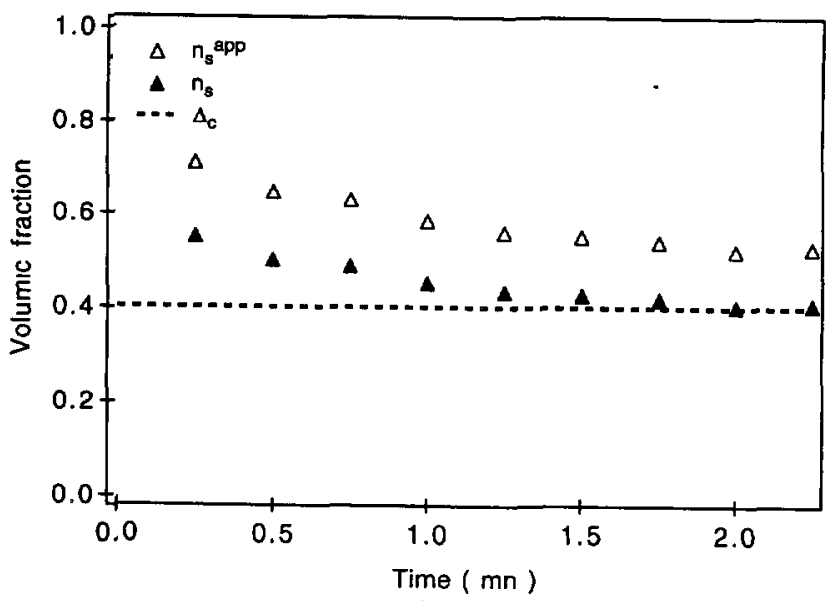

c)

Fig. 6. - a) Radius of the circular envelope of the germ of figure 5 versus time $\left(\Delta_{c}=0.4\right.$, $d=9.2 \mu \mathrm{m}$ ). b) Its surface area versus time. c) The apparent and real volumic fractions of solid inside the hexagonal envelope of the germ. Note that $n$, tends to $\Delta_{c}$ contrary to $n_{s}^{\text {app }}$ which is larger. 


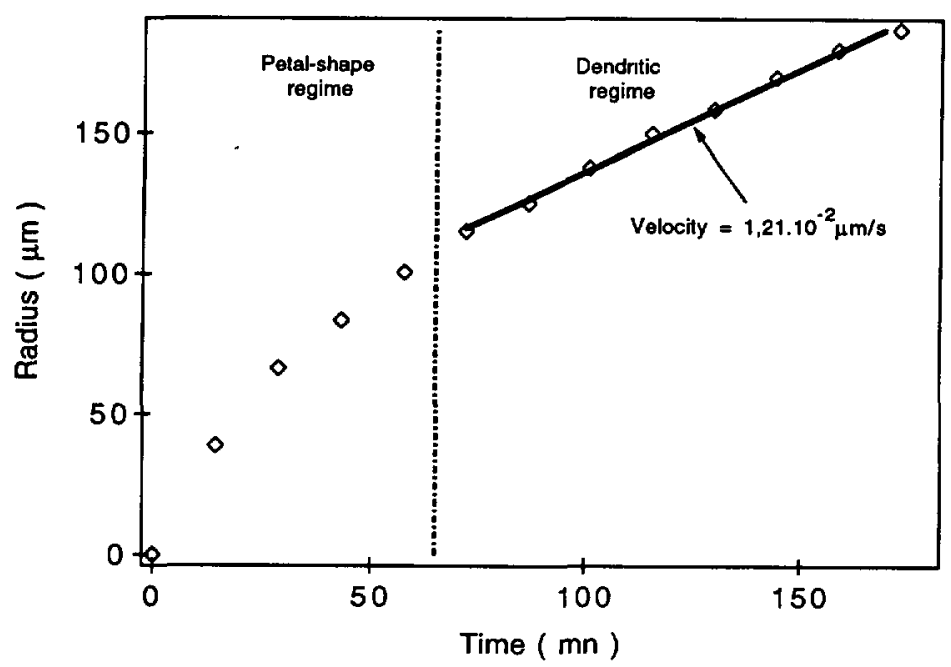

a)

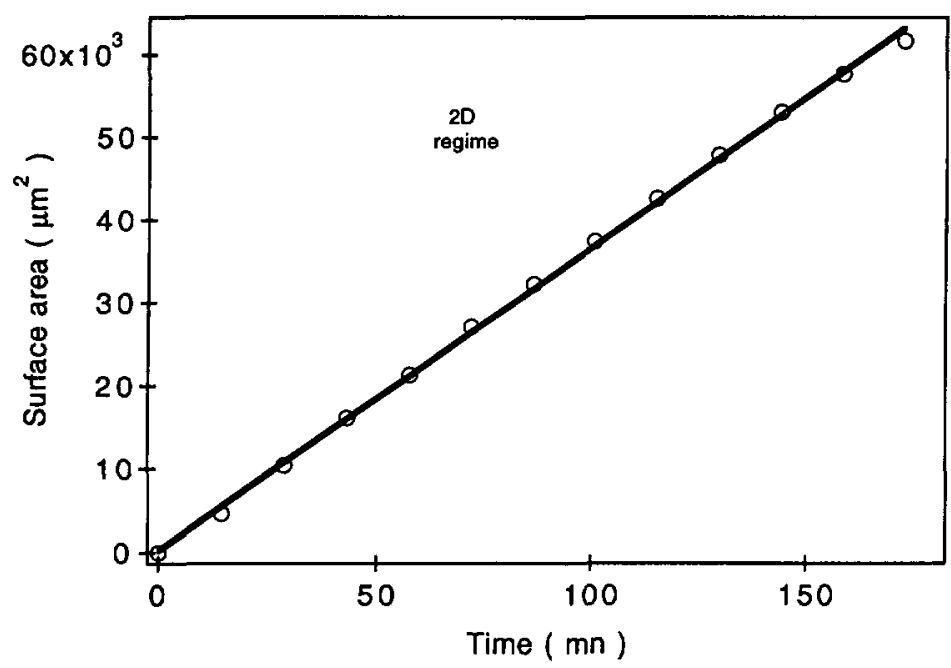

b)

Fig. 7. - a) Radius of the circular envelope of a germ growing at $\Delta_{c}=0.1$ in a $2 \mu$ m-thick sample. b) Its surface area versus time.

4.1 TWO-DIMENSIONAL DENDRITES. - These dendrites have already been studied previously $[5 a, 6]$. In this subsection we show that their properties do not depend on the sample thickness even if they are not strickly $2 \mathrm{D}$ because of the presence of a meniscus in the sample thickness. Indeed, the liquid partly wets the glass plates with an equilibrium contact angle close to $30^{\circ}[9]$.

In figure 9, we have plotted their stability constant $\sigma=2 D_{\mathrm{L}} d_{0}^{\mathcal{C}} / \rho^{2} V$ as a function of the sample thickness. Within the experimental error, this quantity is constant and equals $\sigma_{2 \mathrm{D}}^{*}=0.041 \pm 0.01$. This value is in good agreement with that we found previously $\left(\sigma_{2 \mathrm{D}}^{*}=0.039,[5 \mathrm{a}]\right)$ but is larger than the theoretical value calculated by Ben Amar by taking 


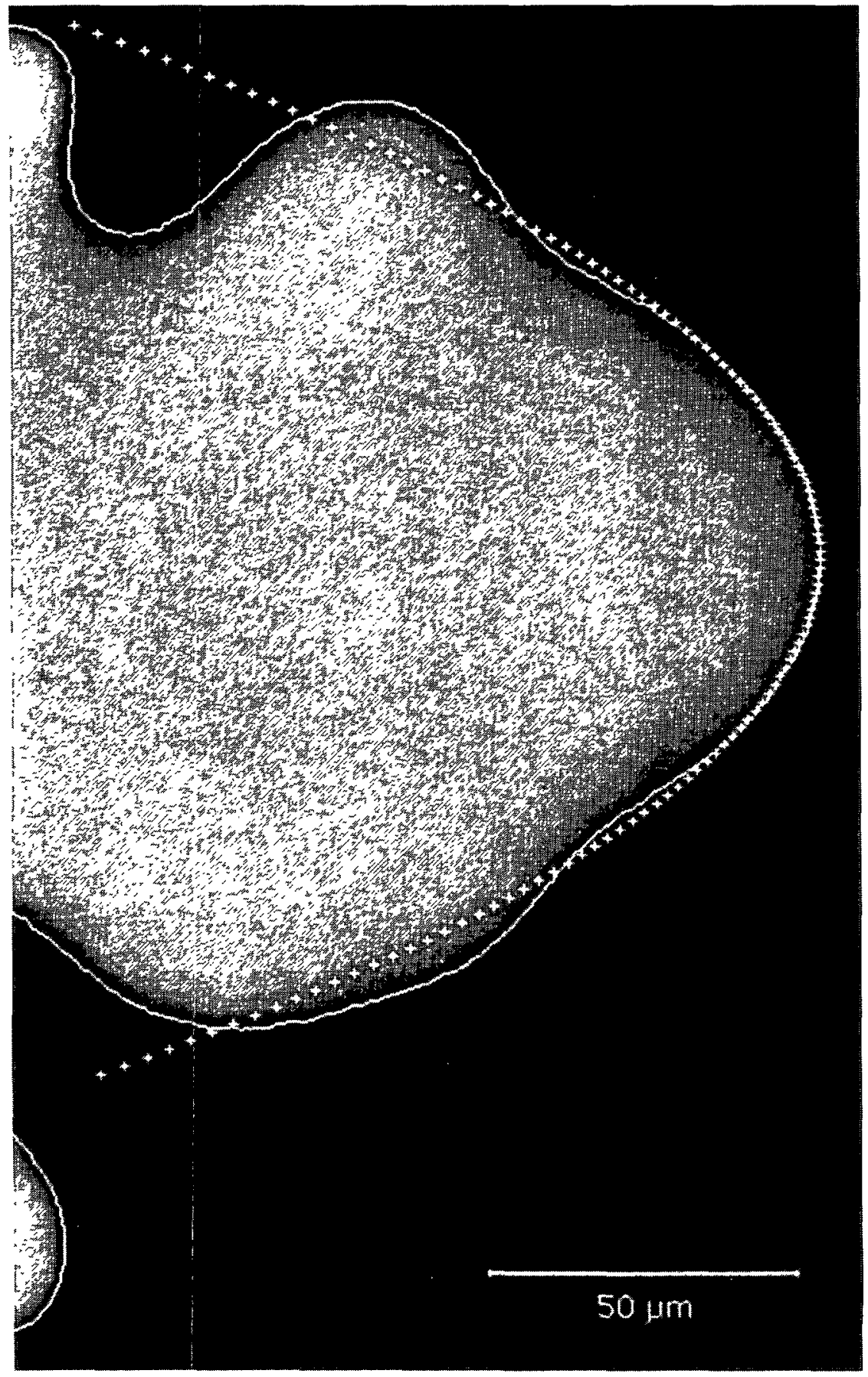

Fig. 8. - Best parabolıc fit of the tip of a two-dimensional dendrite. 


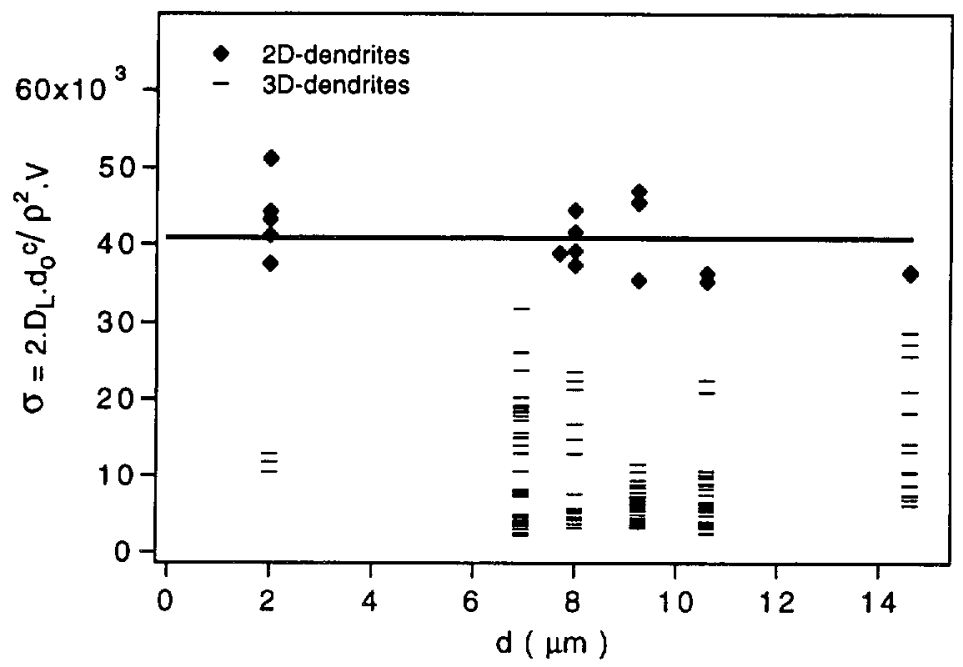

Fig. 9. - Stability constant $\sigma$ as a function of the sample thickness $d$ for 2D- and 3D-dendrites.

$\varepsilon_{6}=2.6 \times 10^{-3}[8]$ and $D_{\mathrm{S}} / D_{\mathrm{L}}=0.6[9]: \sigma_{2 \mathrm{D}}^{*}$ (theory) $=0.033\left({ }^{1}\right)$. This theory-experiment discrepancy could be due to meniscus effects but we did not observe a clear dependence on the sample thickness. Moreover, this discrepancy is often observed in 3D-experiments [2] where there is no meniscus so that we believe that it could rather be explained by the presence of experimental noise. Indeed, Brener and Ben Amar [11] have recently shown that any perturbation tends to increase the value of $\sigma$. We also found interesting to plot in figure 10 the stability constant as a function of the ratio $\ell_{\mathrm{d}} / d$ of the diffusion length over the thickness. This graph shows that $P_{\mathrm{d}} / d>50$ for all $2 \mathrm{D}$-dendrites. This inequality is strong and shows the difficulty to make $2 \mathrm{D}$-dendrites at large supersaturation.

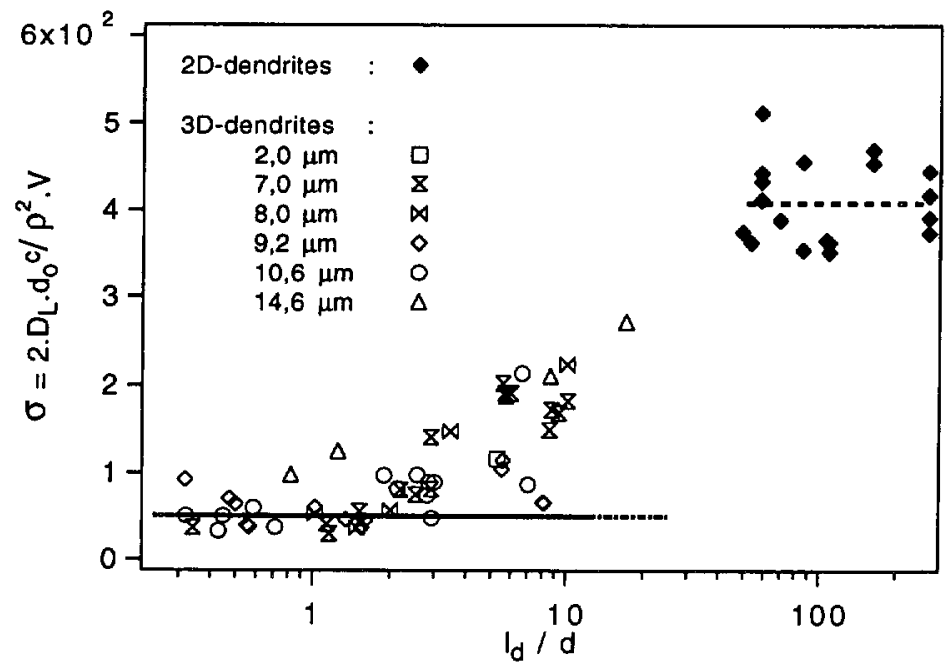

Fig. 10. - Stability constant $\sigma$ as a function of the ratio $\ell_{\mathrm{d}} / d$ for 2D- and 3D-dendrites.

(1) This value is different from that given in reference [8] calculated by takıng $D_{\checkmark} / D_{\mathrm{L}}=0.3$. New experiments of directional solidification [9] have given $D, / D_{\mathrm{L}}=0.6$. 
Finally, we also checked (Fig. 11) that these dendrites follow the two-dimensional Ivantsov's law relating the Péclet number $P=\rho V / 2 D_{\mathrm{L}}$ to the supersatuation $\Delta$. We recall that this relation reads :

$$
\Delta_{\mathrm{c}}=2 \sqrt{P} \exp (P) \int_{\sqrt{P}}^{+\infty} \exp \left(-s^{2}\right) \mathrm{d} s .
$$

It is represented in figure 11 by the solid curve.

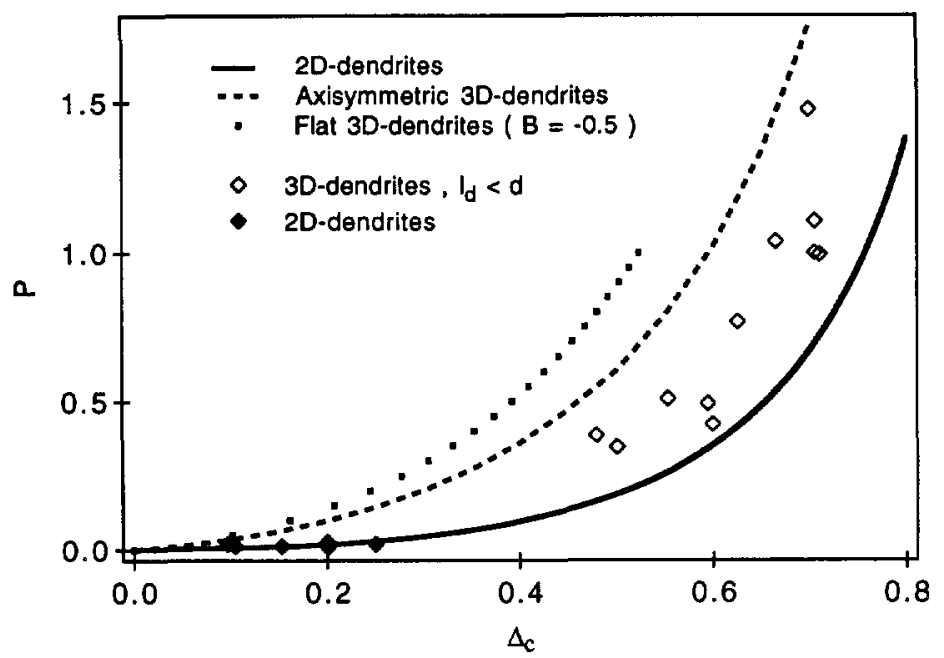

Fig. 11. - Péclet number $P$ as a function of the supersaturation $\Delta_{\mathrm{c}}$. The three curves are theoretical and have been calculated from equation (3) for 2D-dendrites (solid curve), from equation (4) for axisymmetric 3D-dendrites (dashed curve) and from equation (6) with $B=-0.5$ for flat 3D-dendrites (dotted curve). This last curve corresponds to dendrites with a radius of curvature twice as small in the sample thickness as in the horizontal plane.

4.2 THREE-DIMENSIONAL DENDRITES. - We carried out similar experiments on these dendrites.

Let us first discuss their stability constant. This quantity is plotted in figures 9 and 10 respectively as a function of the sample thickness and the ratio $\ell_{\mathrm{d}} / d$. It clearly appears that $\sigma$ strongly depends on the thickness as long as $\ell_{\mathrm{d}} / d>1$. By contrast, $\sigma$ tends to a constant $\sigma_{3 \mathrm{D}}^{*} \approx 0.005 \pm 0.002$ when $\ell_{\mathrm{d}} / d<1$. This value is smaller than that found for $2 \mathrm{D}$-dendrites. This result is in agreement with the observations of Janiaud et al. [4] who found that $\rho^{2} V$ increases with the sample thickness and tends to a constant when $\ell_{\mathrm{d}} / d<1$. Thus, the decrease of $\sigma_{3 \mathrm{D}}^{*}$ can in part be explained by a thickness effect. It could also be due to the large values of the Péclet number $P$. Indeed, in our experiments, $P$ ranges between $7 \times 10^{-2}$ and 1.5 and is much larger than in the usual dendritic growth experiments [2]. Numerical simulations [13] have shown that $\sigma^{*}$ is a strongly decreasing function of the Péclet number $P$; for example, we can expect for $P=1.5$ that $\sigma^{*}$ roughly decreases by a factor 2 in the case of 3Daxisymmetric dendrites. On the other hand, we do not understand why $\sigma_{3 \mathrm{D}}^{*}$ tends to a well defined constant for $\ell_{\mathrm{d}} / d<1$.

We also emphasize that $\ell_{\mathrm{d}} / d<10$ for all the observed 3D-dendrites whereas $\ell_{\mathrm{d}} / d>50$ for the $2 \mathrm{D}$-dendrites. This means that there is a jump in velocity at the supersaturation $\Delta_{\mathrm{c}}^{*}$ for which $2 \mathrm{D}$-dendrites transform into $3 \mathrm{D}$-dendrites. 
To go farther, we also tried to verify the 3D-Ivantsov's relation. If the dendrite is axisymmetric, this relation reads :

$$
\Delta_{\mathrm{c}}=P \exp (P) \int_{P}^{+\infty} \frac{\exp (-s)}{s} \mathrm{~d} s .
$$

In figure 11, we plotted this function (dashed curve) and we reported the experimental points corresponding to dendrites for which $\ell_{\mathrm{d}} / d<1$ (only these dendrites have a well-defined stability constant). These points lie systematically below the theoretical curve corresponding to 3D axisymmetric dendrites. This might be due to their flattening in the horizontal plane, since, in general, $\rho>d / 2$. We thus tried to fit the tip of our dendrites by an elliptic paraboloid of equation

$$
x^{2}+\frac{z^{2}}{B+1}=-2 \rho y
$$

where $\rho$ is the tip radius of curvature in the horizontal $(x, y)$ plane and $B$ a parameter characterizing the eccentricity of the elliptic section in a vertical plane perpendicular to the growth direction. We know, from the Horvay and Cahn calculations [12], that such a paraboloid is a possible solution to the growth equations. In this case, the Ivantsov relation becomes

$$
\Delta_{\mathrm{c}}=P \sqrt{1+B} \exp (P) \int_{P}^{+\infty} \frac{\exp (-s) \mathrm{d} s}{\sqrt{s(s+P B)}}
$$

where $P$ is the Péclet number calculated with the radius of curvature in the $(x, y)$ plane. Experimentally, $B<0$ since $\rho>d / 2$. In figure 11, we plotted theoretical law (6) for $B=-0.5$ (dotted curve). This curve is above that corresponding to axisymmetric dendrites. Consequently, it is impossible to explain the experimental results in this way.

Kinetic effects may be relevant to explain the discrepancy between theoretical and experimental Péclet numbers. Indeed, the fastest dendrites grow with velocities of a few microns per second. In the case of the hexaoctyloxytriphenylene, the kinetic coefficient has been measured and found to be equal to $\mu=130 \mu \mathrm{m} / \mathrm{s} / \mathrm{K}$ [7]. If kinetic effects tend to decrease the growth velocity [13], it remains difficult to compare our experiments to the existing theoretical results because of the non-axisymmetry of the observed dendrites.

\section{Concluding remarks.}

We have shown that two types of stationary dendrites exist depending on the values of the supersaturation $\Delta$ and the ratio $\mathcal{P}_{\mathrm{d}} / d$.

If $\Delta$ is small $\left(\Delta_{\mathrm{c}}<\Delta_{\mathrm{c}}^{*} \approx 0.3-0.5\right)$ and as long as $\ell_{\mathrm{d}} / d>50$, the dendrites fill the whole sample thickness and can be considered as two-dimensional. In spite of the presence of a meniscus, they satisfy the 2D Ivantsov relation and have a well-defined stability constant which is independent of the sample thickness $\left(\sigma_{2 D}^{*} \approx 0.041\right)$. As in many other materials, the value of this constant is larger than that calculated theoretically [2]. This disagreement is currently ascribed to experimental noise.

At large supersaturation $\left(\Delta_{\mathrm{c}}>\Delta_{\mathrm{c}}^{*}\right)$, dendrites separate from the glass plates and become three-dimensional. Then they grow faster than $2 \mathrm{D}$-dendrites since $\boldsymbol{\ell}_{\mathrm{d}} / d<10$. In general, it is not possible to attribute to them a well-defined stability constant, except at large enough supersaturation when $\ell_{\mathrm{d}} / d<1\left(\sigma_{3 \mathrm{D}}^{*} \approx 0.005\right)$. On the other hand, the 3D-Ivantsov relation for free dendrites is not fulfilled, even when the flattening of the tip region is taken into account (in 
this case, the disagreement is still larger). Even if Péclet number and kinetic effects may in part explain the behaviour of both $\sigma^{*}$ and $P$ when $\Delta_{c}$ increases, we think that the dendrites are not free and still feel the confinement effects between the two glass plates, even when $\ell_{\mathrm{d}} \approx d / 3$. Consequently, it is not enough that $\sigma$ be constant and $\ell_{\mathrm{d}}<d$, to assert that a dendrite is free.

\section{Acknowledgments.}

This work was supported by C.N.E.S. contract n 93/02.15. We thank J. Malthête for kindly synthesizing the discotic liquid crystal and D. Temkin for fruitful discussions.

\section{References}

[1] Meiron D. I., Phys Rev A 33 (1986) 2704 ;

Kessler D. A., Koplik J., Levine H., Phys. Rev. A 33 (1986) 3352.

Ben Amar M., Pomeau Y., Europhys Lett. 2 (1986) 307.

Brener E. A., Mel'nikov V. I., Adv. Phys. 40 (1991) 53.

[2] Gollub J. P., in Growth and Form, M. Ben Amar et al., Eds. (Plenum Press, New York. 1991) p. 45 ;

Mushol M., Liu D., Cummins H. Z., Phys. Rev. A 46 (1992) 1038.

[3] Ben Amar M., Brener E., Phys. Rev. Lett. 71 (1993) 589.

[4] Janiaud B., Bouissou Ph., Perrin B., Tabeling P., Phys. Rev. A 41 (1990) 7059.

[5] a) Oswald P., J. Phys. France 49 (1988) 1083 ; b) 49 (1988) 2119 ; c) J. Phys. Colloq. France 50 (1989) C3-127.

[6] Oswald P., Malthête J., Pelcé P., J. Phys. France 50 (1989) 2121.

[7] Géminard J. C., Oswald P., Temkin D., Malthête J., Europhys. Lett. 22 (1993) 66.

[8] Oswald P., J. Phys. France 49 (1988) 1083.

[9] Géminard J. C., Thèse de doctorat, Université Claude Bernard Lyon I, 1993.

[10] Brush L. N., Sekerka R. F., J. Cryst. Growth 96 (1989) 419.

[11] Ben Amar M., Brener E., Phys. Rev. E 47 (1992) 534.

[12] Horvay G., Cahn J. W., Acta Met. 9 (1961) 695.

[13] Ben Amar M., Phys. Rev. A 41 (1990) 2080. 